\title{
TRAIN TIMETABLE RESCHEDULING GENERATION BASED ON VEHICLE TYPE AND TRAIN ROUTE COMBINATIONS
}

\author{
T. KATORI \& T. IZUMI \\ Nihon University, Japan.
}

\begin{abstract}
Train rescheduling means a transient situation to correct a train diagram in a suspended state due to traffic accidents or disasters. Automatic (or half manual) rescheduling has been studied, and such previous research has shown promising results.

In this paper, we describe a train timetable rescheduling method. In Japanese urban areas, some private companies operate on each other's tracks. If vehicle types have limitations due to ground facilities or company rules, the vehicle has to be operated under these limitations even when running on a rescheduled timetable. Even if the ground facilities of different companies have uniform conditions, local and rapid trains must be operated in a distinct manner.

Therefore, we suggest a rescheduling method. With this method, each vehicle type and its vehicle routes based on the track layout are registered, and rescheduling diagrams are composed with the route combinations.

Important conditions to decide the combinations are vehicle location at the operation resumption time and the introduction of same-type vehicles at an originally unscheduled timing. We compare the traffic effects for some combinations of the latter situation where originally unscheduled same-type vehicles are introduced for rescheduling. The evaluation values are average headway time and its standard deviation at all stations on the timetable.

We apply our rescheduling method to a theoretical line and timetable modelled on existing urban lines in Japan where trains go and come back on double tracks, and indicate the efficacy of our rescheduling method.

Keywords: route combinations, train rescheduling, vehicle type.
\end{abstract}

\section{INTRODUCTION}

Railway transport is generally considered safe and punctual, but sometimes the transport cannot operate on the scheduled timetable due to traffic accidents or disasters. Train rescheduling means a transient situation to correct a suspended state. When a traffic accident suddenly happens, a rescheduling timetable has to be generated quickly, within 5 to $10 \mathrm{~min}$. Train rescheduling means swapping the departure order and changing the turning or terminal station. Here, a short delay of a few minutes which can be solved by a shorter turning time at the terminal station is not included.

In Japan, a rescheduling timetable used to be generated by expert operators, but the number of operators was decreased due to management streamlining, and now this work has to be processed automatically. Computer processor performance improved and processors are able to solve realistic size problems $[1,2]$. Many railway companies use programmed traffic control systems, and the timetable data are kept and processed on such computer systems. Therefore, automatic rescheduling became fit for computers, because manual converting is not needed [3]. 


\section{COMPRAIL}

However, companies running on through other company facilities to accommodate passengers face issues such as running area restrictions for different vehicle types. This limitation makes rescheduling difficult [4].

In this paper, we describe how to generate train rescheduling timetables considering vehicle-type operation. The rescheduled timetable has to satisfy two limitations, namely certain track areas are bound to certain vehicle types, and trains cannot be positioned at the same location at the same time. To solve these limitations, the track layout is managed by dividing the time interval system into data blocks. Train routes are registered based on the tracks between the start and terminal stations based on running time. The rescheduling timetable is composed with combinations of these routes.

This rescheduling procedure has four parameters: traffic accident happening time, communication time for all trains, resumption time and complete recovery time. We compare some rescheduled timetables based on variations of the above parameters. In this paper, we adopt only turning operation on double tracks as is usual in Japanese urban areas.

\section{ASSUMPTIONS AND DATA TYPES FOR GENERATING TRAIN RESCHEDULING}

\subsection{Assumptions for generating train rescheduling}

Train rescheduling timetables are generated under the following assumptions and modelling:

- Train operation unit time is $30 \mathrm{~s}$. This means train locations are renewed every $30 \mathrm{~s}$.

- Minimum headway time between trains is 2 min.

- Minimum dwell time is $5 \mathrm{~min}$ at turning operation and $30 \mathrm{~s}$ at the other middle stations.

- Running time between stations is the same on the scheduled timetable as on the rescheduling timetable. The train stops at the station only, does not stop between stations and does not operate at slow speed. In reality, trains may run at a slower speed than normal, but on the rescheduling plan, trains run on standard operation time only.

- All vehicles have the same driving performance. (On Japanese urban lines, most trains use the same vehicle type.)

- Trains are never partitioned or combined.

\subsection{Data types and format}

\subsubsection{Track layout data}

The track layout is divided by data blocks based on running time, and the blocks have ID numbers. In this study, the unit time is $30 \mathrm{~s}$ as indicated in Section 2.1. When the running time between stations is $2 \mathrm{~min}$, this area is divided into four blocks. For crossover, $30 \mathrm{~s}$ are allotted, even if the train can run the distance in a shorter time. So, the track layout is expressed by blocks considering both time and distance. These block sections differ from real signalling sections, because this model is a theoretically simple model. If the unit time is shortened by 1 or $5 \mathrm{~s}$, the sections can express a more detailed solution than signalling sections.

Figure 1 shows an example of a block division for a sample track layout and the data concerning the block number and the location from the terminal. 


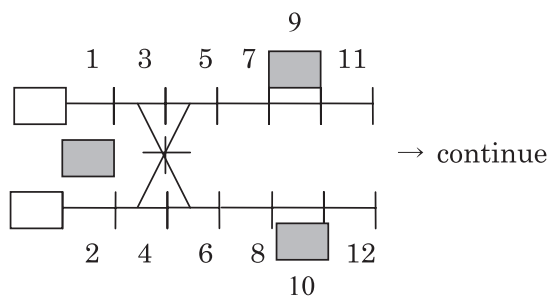

Figure 1: An example of a block division for track layout. (

: platform).

Table 1: Data form for a train schedule.

\begin{tabular}{cccccccccc}
\hline Vehicle type & $\begin{array}{c}\text { Time (min) } \\
\text { Train No.l }\end{array}$ & 0 & 0.5 & 1 & 1.5 & 2 & 2.5 & \\
\hline 1 & 0 & 1 & 3 & 5 & 7 & 9 & 9 & $\ldots$ \\
2 & 1 & 16 & 14 & 12 & 10 & 10 & 8 & $\ldots$ \\
1 & 2 & 61 & 59 & 58 & 56 & 54 & 54 & $\ldots$ \\
2 & 3 & 63 & 63 & 63 & 65 & 67 & 67 & $\ldots$ \\
\hline
\end{tabular}

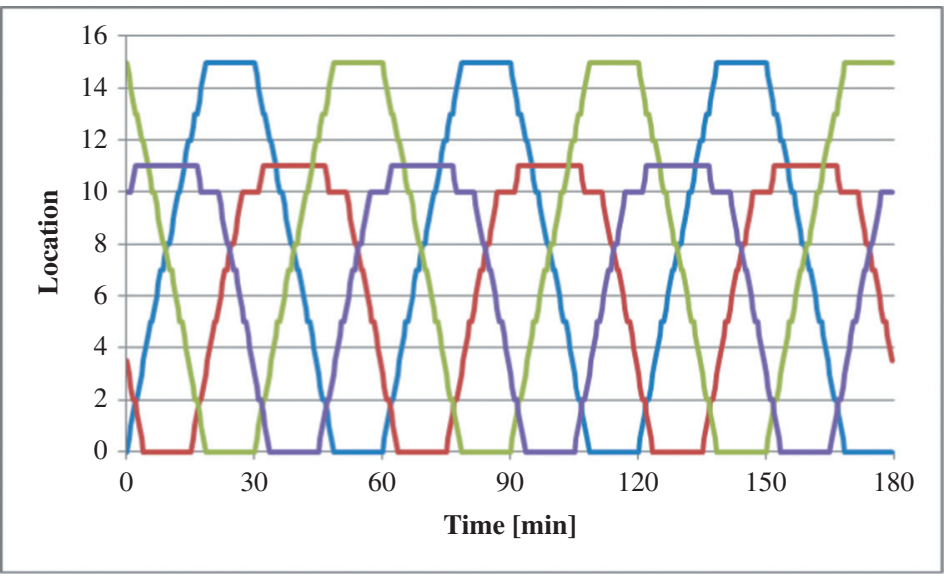

Figure 2: Sample of a train schedule diagram.

\subsubsection{Train diagram data}

Train diagram data express a row of track layout block numbers and train location versus time. Each vehicle operates differently, and the vehicles continue to have location data after turning at the terminal station.

Table 1 shows a data form for a train schedule, and Fig. 2 is a train diagram based on Table 1.

\subsubsection{Route data}

Route data are already combined in the scheduled operation. Therefore, these data are only needed for rescheduling. 


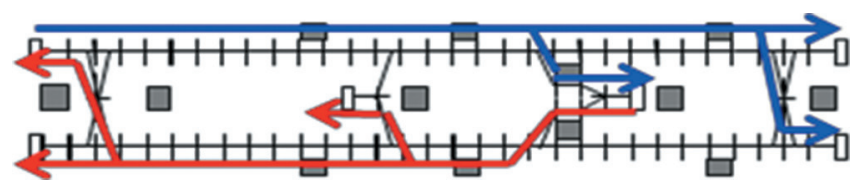

Figure 3: Some examples of route sets.

Route data contain information about possible routes, required time and possible vehicles for a particular route between the start and terminal stations.

Figure 3 shows some examples of route sets. Blue arrows indicate routes from the upper left track, and the red ones from the middle right track. All possible routes are registered per vehicle type, between all turning tracks.

\section{HOW TO GENERATE A TRAIN RESCHEDULING TIMETABLE CONSIDERING VEHICLE TYPES AND ROUTE COMBINATIONS}

\subsection{Parameters for rescheduling}

The following four parameters have to be provided to generate a timetable:

- time and location of the traffic accident

- time needed to communicate the traffic accident to all trains

- resumption time

- complete recovery time.

Figure 4 shows these parameters on the train diagram.

The time needed to communicate the traffic accident to all trains means the delay that is required till all other trains are informed. The train in the accident stops soon, but other trains continue to run on the scheduled timetable until they receive the accident information. All trains stop after this communication time.

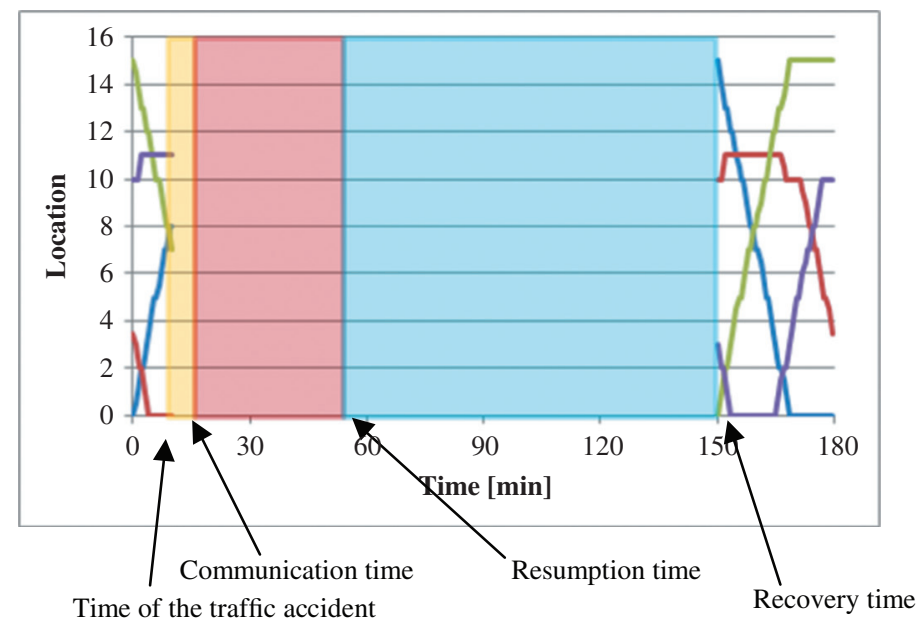

Figure 4: Rescheduling parameters. 
Resumption time can set different times for the accident area and different areas and considers turning operation on the part of the line.

Complete recovery time means the end of the rescheduling operation time. The rescheduling timetable is generated between the resumption and complete recovery time.

\subsection{Rescheduling timetable generation}

\subsubsection{Pre-processing}

All trains stop at the accident time or the communication time. The stopping location is the nearest stopping station for each train.

Trains are operated by the rescheduling timetable after the resumption time.

First, new destination stations are redefined for all trains. As an experimental rule, the new destination is the original destination of the train, but if there are no tracks available when the train is to reach its original destination, the destination station changes to a closer station with a possibility to turn. If the new destination is closer than original one with a possibility to turn, trains cannot operate because the number of turning equipment is less than the number of trains. The decision of a new destination station is a very important condition, because this condition defines the rescheduling solution quality.

Also, each train is not necessarily located at its start station on the scheduled timetable at the complete recovery time. Therefore, the origin station and starting time are defined for running trains at recovery time.

Figure 5 shows pre-processed results for Fig. 4. Train diagrams are added until the destination station after the resumption time and from the origin station before complete recovery time.

A rescheduling timetable is calculated between the new origin station at the starting time and the destination at the complete recovery time for each train. This time is called rescheduling time.

\subsubsection{Vehicle type and operation}

If each vehicle is not on the location of the original scheduling diagram at the complete recovery time, there is no problem if a vehicle of the same type operates at that location and direction. Therefore, same vehicle-type connection at the complete recovery time is also an important condition.

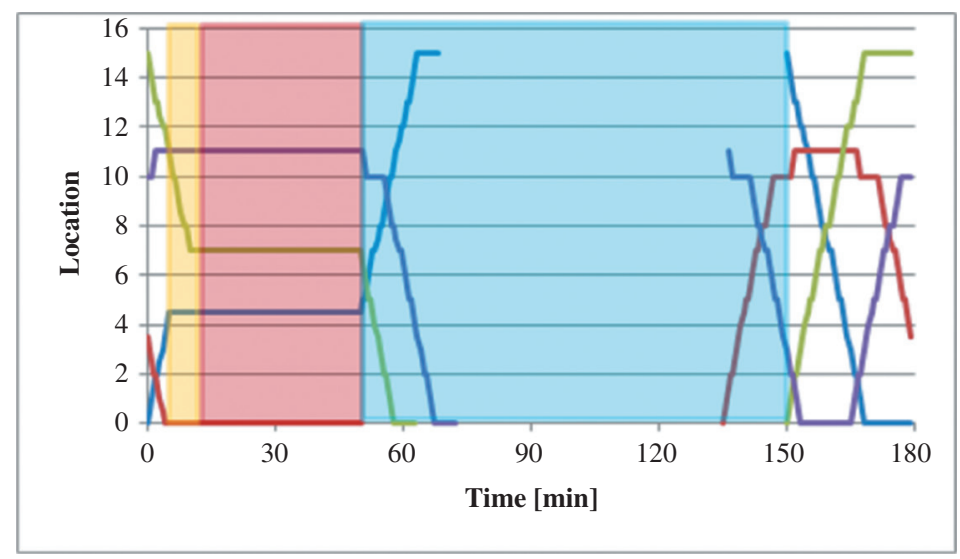

Figure 5: A result of pre-processing. 


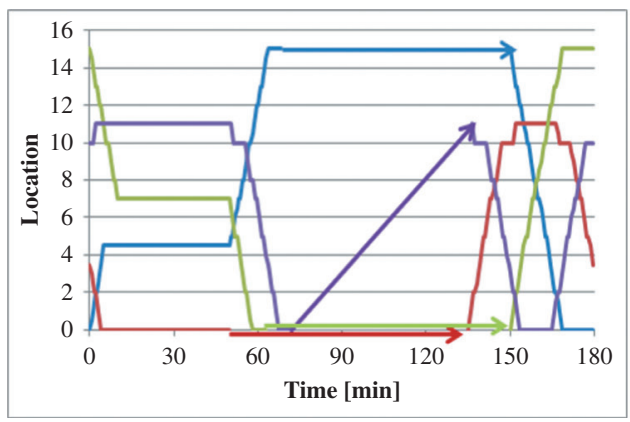

(a) No vehicle change

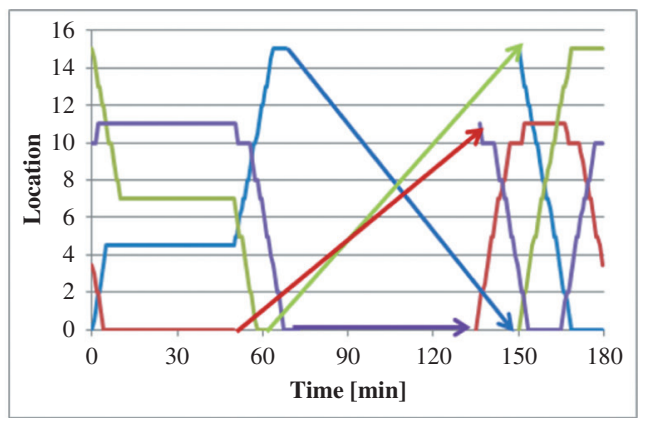

(b) Vehicle change

Figure 6: Connection for vehicle type on operation.

In this paper, this is also set as a parameter, and the vehicle connection effect is compared.

Figure 6 shows some connections for vehicle operation. Figure 6(a) indicates the trains connecting with the original operation, and Fig. 6(b) indicates a substitution between same-type vehicles.

\subsubsection{Route generation}

All possible train routes between any turning station during rescheduling time are investigated. For this procedure, route data in Section 2.2.3 are used, and all possible route combinations are investigated recursively and saved. This investigation uses the start and terminal track numbers and required time. The total required time should be shorter than the rescheduling one. This investigation can be considered a knapsack problem with a limitation, where the terminal track number $g_{i}$ in route $R_{i}$ must be the start track number $s_{i+1}$ in next route $R_{i+1}$.

If the rescheduling time is rtime, this relation gives the inequality

$$
\Sigma R_{i}<\text { rtime }
$$

Because the route data include the possible vehicle types for a specific route, only route combinations for possible areas are generated. Routes are searched for all running trains.

Figure 7 shows an example of some searched routes. Numbers 1-4 in the figure are routes on the diagram from $t=68 \mathrm{~min}$, location number 15 to $t=150 \mathrm{~min}$, same location number. Similar routes are searched for other trains.

\subsubsection{Rescheduling timetable generation}

A rescheduling timetable is generated with all obtained route combinations for all trains. These combinations include the case of more than one train at the same time and on the same track. Such case is rejected as an impossible operation solution. For each train $i$ and $j$, location $L$ in time $t$, if the following equation gives

$$
L_{i t}=L_{j t} \quad(i \neq j),
$$

then a backtrack procedure is adopted, and computation time decreases. This way, crossing hindrance is also avoided. When trains are not located on the same track, the route combinations that do not maintain the minimum headway time are rejected. 


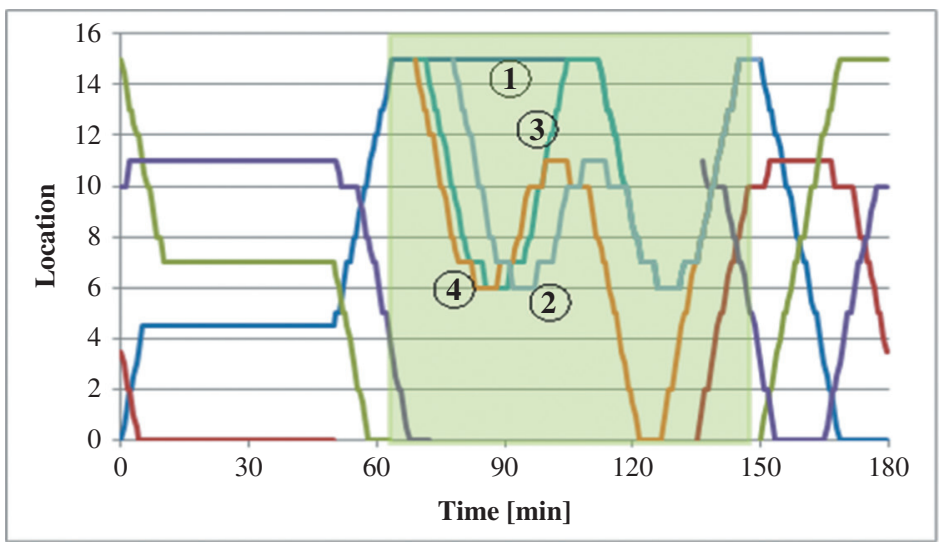

Figure 7: A route tree example (from terminal to terminal).

\subsubsection{Evaluation value}

The quality of the generated rescheduling timetable is evaluated, and the best solution is adopted. The evaluation value is the total product at station $i$ on average headway time $a v e_{i}$ and standard deviation $s_{i}$ for the same operating direction. A shorter average headway time at any station results in a large number of trains operations, which is convenient for the passenger. Additionally, a smaller standard deviation at the station means a uniform density operation. Both the smaller elements are good, and the evaluation value is given in eqn (3).

$$
E=\Sigma\left(\text { ave }_{i} * s_{i}\right)\left(\min ^{2}\right)
$$

The shorter the average headway time becomes, the higher the number of train, but distribution per an hour cannot be decided only by this. Therefore, standard deviation is used to uniformly distribute the train headway time and ensure even distribution of passenger numbers on the trains.

Under the current combination of parameters, several rescheduling timetables are possible. However, the smallest evaluation value is adopted as the solution.

\section{RESULTS AND DISCUSSION}

\subsection{Model line for this study}

The rescheduling procedure is applied to a line modelled on existing urban lines, and a rescheduling timetable is generated.

Figure 8 shows the line modelled on existing lines, and Table 2 shows the conditions of the line under consideration.

On this model line, two vehicle types operate. One type can operate on the whole line, and the other can run between station numbers 0 and 5. This condition means, for example, that direct current is available between station numbers 0 and 5, but alternative current or diesel operation is used between station numbers 5 and 8 .

Figure 9 (= Fig. 2) shows the scheduled diagram for the model line. Blue and green vehicle types can run on the whole line, but the red and purple ones are limited and can only run until the middle turning station. Only local trains operate on this line. Trains running till station 5 and trains running till station 8 operate alternatively every $15 \mathrm{~min}$. A rescheduling timetable is generated for this line and diagram by changing the parameters. 


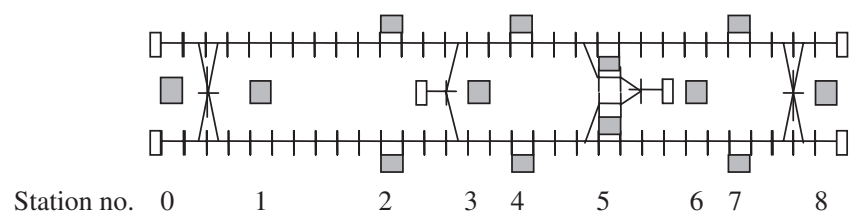

Figure 8: Theoretical line modelled on existing line ( : station).

Table 2: Conditions of the line under consideration.

\begin{tabular}{lc}
\hline Number of stations & 9 stations \\
Running time (non-stop) & $15 \mathrm{~min}$ \\
Total number of blocks & 68 \\
Number of routes & 18 \\
Total length & $16.2 \mathrm{~km}$ \\
\hline
\end{tabular}

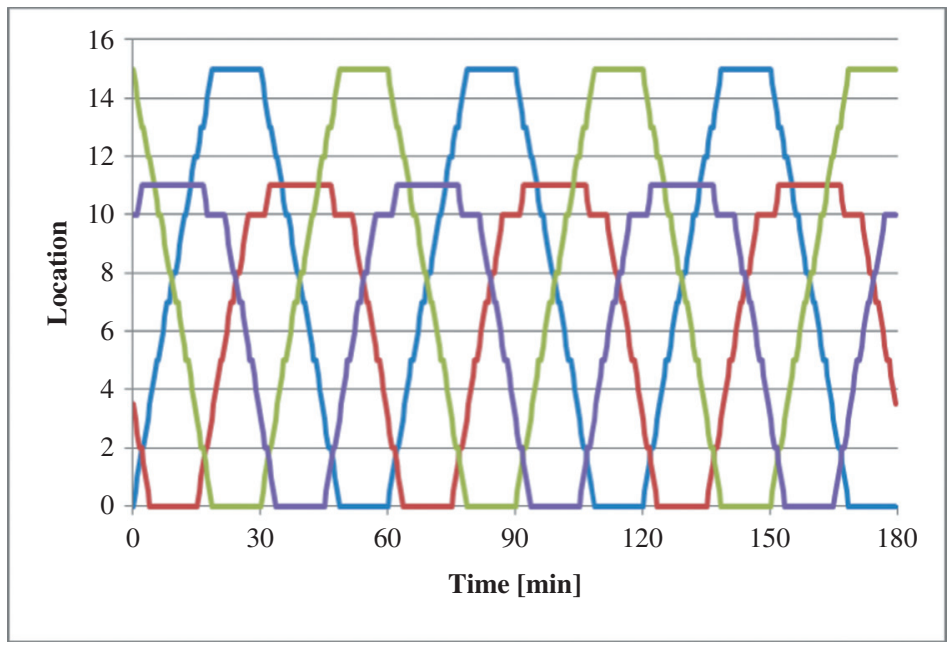

Figure 9: Originally scheduled train diagram (= Fig. 2).

\subsection{Results}

On the scheduled timetable, a traffic accident happens 5 min after the train departure. Communication time is set to take $5 \mathrm{~min}$, resumption time is $40 \mathrm{~min}$, and complete recovery operation time is set to vary. All parameter conditions and their evaluation values are shown in Table 3 .

Figures 10(a), 10(b) and 10(e) are similar to Fig. 6(a), i.e. they connect to the original vehicle operation, and Figs. 10(c), 10(d) and 10(f) are similar to Fig. 6(b), i.e. they connect to same-type vehicles in different operations. In addition, Figs. 10(e) and 10(f) indicate waiting solutions where the train waits until the cycle repeats. These solutions can possibly not be called rescheduling. For Figs. 10(e) and 10(f), the resumption time is set at 30 min because setting it at $40 \mathrm{~min}$ resulted in the same solutions.

Figure 10 shows a sample of the rescheduled diagram under these conditions. 


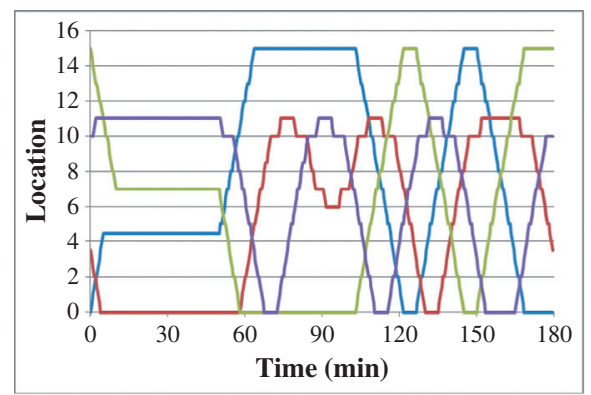

(a) Rescheduling without vehicle change. $($ Recovery time $=150(\mathrm{~min}))$

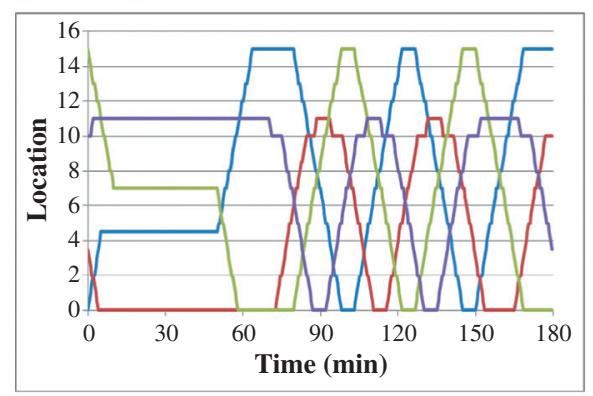

(c) Rescheduling without vehicle change. $($ Recovery time $=150(\min ))$

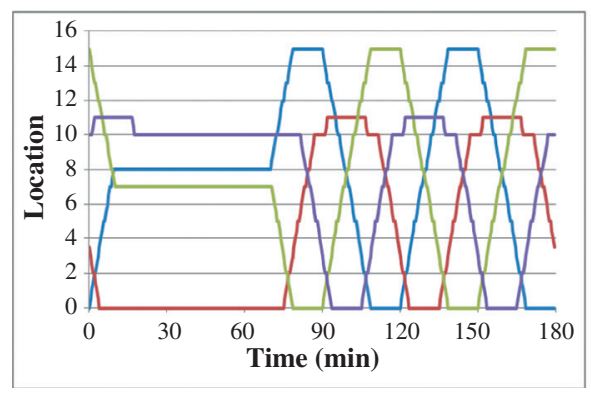

(e) Discontinuation without vehicle change until the cycle is repeated.

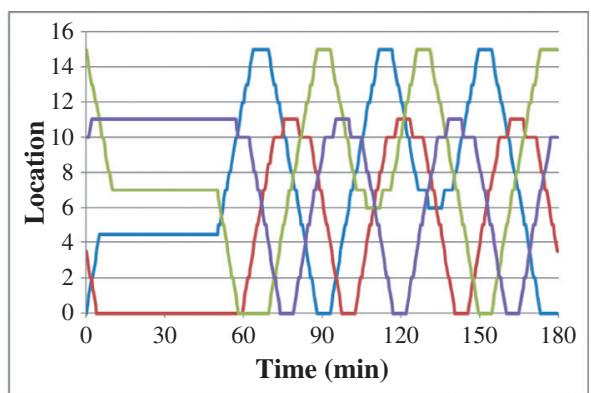

(b) Rescheduling without vehicle change. $($ Recovery time $=178(\min ))$

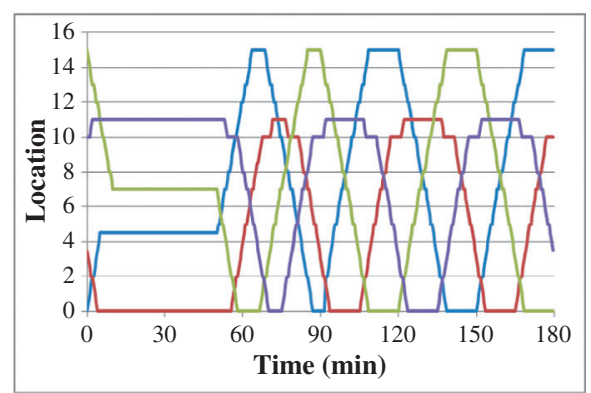

(d) Rescheduling without vehicle change. $($ Recovery time $=95(\mathrm{~min}))$

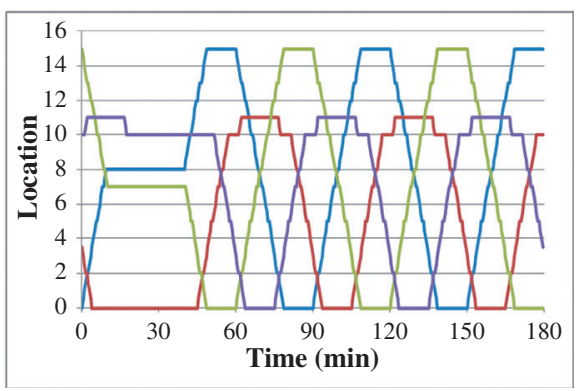

(f) Discontinuation with vehicle change until the cycle is repeated.

Figure 10: Generated diagrams and conditions.

\subsection{Discussion and considering}

\subsubsection{Discussion}

Figures 10(a) and 10(b) show rescheduling without changing vehicle operation. In the diagram, any trains turn at station no. 3, which is generally called the 'mountain cut', and the diagrams give a tricky impression. Figures 10 (b) and 10(d) have the smallest evaluation value based on optimum recovery time. The solution of Fig. 10(d) is obtained by only shortening the turning time at the terminal stations. When comparing Figs. 10(a) and 10(b) with Figs. 10(c) and 10(d) in terms of flexible operation effect for the same vehicle type, Figs. 10(c) and 10(d) have smaller evaluation values. For operation with change vehicle, 
Table 3: Parameter conditions for train rescheduling.

\begin{tabular}{clcccc}
\hline & Method & $\begin{array}{c}\text { Resumption } \\
\text { time }(\mathrm{min})\end{array}$ & $\begin{array}{c}\text { Recovery time } \\
(\mathrm{min})\end{array}$ & $\begin{array}{c}\text { Connection } \\
\text { type }\end{array}$ & $\begin{array}{c}\text { Evaluation value } \\
\left(\mathrm{min}^{2}\right)\end{array}$ \\
\hline a & Rescheduling & 40 & 150 & No change & 5,772 \\
b & Rescheduling & 40 & 178 & No change & 1,400 \\
c & Rescheduling & 40 & 150 & Change & 2,258 \\
d & Rescheduling & 40 & 95 & Change & 1,391 \\
e & Waiting & 30 & 78 & No change & 4,100 \\
f & Waiting & 30 & 48 & Change & 2,120 \\
\hline
\end{tabular}

the recovery time on Table 3(d) is shortened. Free operation shortens the complete recovery time and improves the evaluation value.

Figures 10(a)-(d) show the generated rescheduling diagrams; however, sometimes there are no solutions due to parameter combinations.

Figure 11 shows the evaluation values versus recovery time. Resumption time is set constant at $40 \mathrm{~min}$.

The blue line indicates that the operation is back to the originally scheduled diagram.

The red line indicates connections to other operations with the same vehicle type. It is generally said that a longer recovery time will result in a good rescheduling diagram. If the vehicle operation remains the same as the original one (Fig. 6(a)), then the longer recovery time leads to a smaller evaluation value. On the other hand, if the vehicle operation is changed while keeping the same vehicle type, then the evaluation value feature is hardly related to the recovery time and does not vary much either.

Figure 11 shows that with a recovery time shorter than $175 \mathrm{~min}$, the vehicle operation should be changed if a cyclic diagram is used on this model line. If the recovery time is shorter than the time, the operation should be changed.

Sometimes there are no solutions available for any recovery time, depending on the timetable cycle and the running time of one turning. The reason is that the recovery time is shorter than one turning time. In this procedure, waiting time is not distributed on turning time but used after resumption time only. Accordingly, the generated rescheduling timetable is a tight one.

Figures 10(e) and 10(f) indicate a solution that only consists of waiting using the repeating cycle timetable. Especially Fig. 10(f) results in an earlier recovery time, thanks to a flexible combination of same vehicle types. However, because both Figs. 10(e) and 10(f) are made to wait after the possible resumption time, the evaluation values are not so favourable.

The computation time is about $20 \mathrm{~s}$ on a general personal computer, because the modelled line size and vehicle numbers are small.

\subsubsection{The difference with the procedure by an expert operator}

The proposed rescheduling procedure is similar for the way of thinking by an expert operator. In other words, the flow to make minor adjustments to decide the number of possible turnings for a given vehicle in a given area till the complete recovery time and to avoid crossing hindrance is similar. However, because waiting time is not included in the turning time, only rigid solutions are obtained, and sometimes there are no solutions, depending on the way the complete recovery time is set. In addition, this procedure does not have typical rule-based knowledge. Therefore, solution-searching efficiency is not so high. 


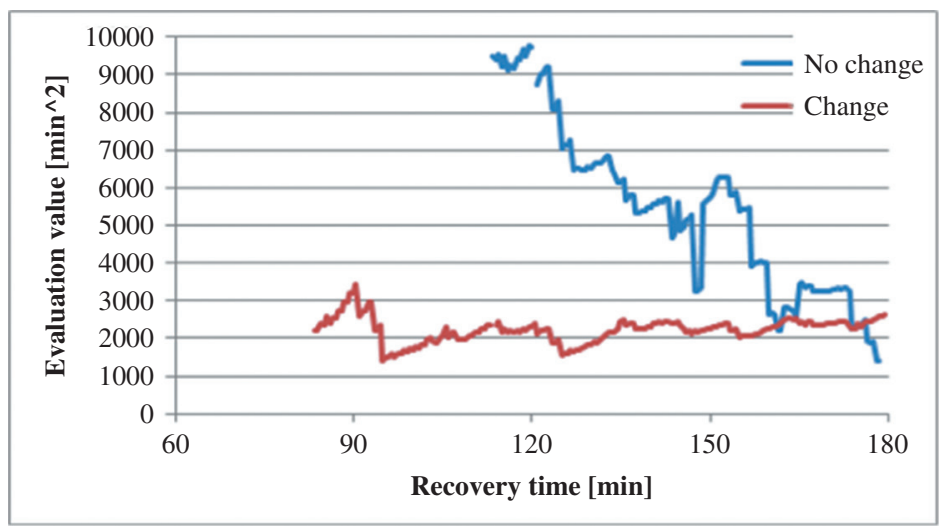

Figure 11: Evaluation values versus recovery time (with or without changing vehicles operation). Resumption time: $40 \mathrm{~min}$.

\section{CONCLUSION}

We suggested how to generate an automatic rescheduling timetable when traffic accidents occur. We considered vehicle types in particular. Possible routes per vehicle type were registered in order to deal with different private companies running through on each other's tracks. The rescheduling timetable is a combination of route combinations including information on vehicle types that can be used.

Important elements for the rescheduling procedure are train locations at the resumption time and how to deal with vehicle operation, i.e. changing or not changing the vehicle operation. Both conditions can still be improved.

In future research, we will focus on automatic determination of vehicle connection and the addition of rapid train operation. In addition, shorter division unit time (track length) can lead to higher accuracy for complex timetables with a larger size. We will also evaluate the method for spider operation as is usual in Europe.

\section{ACKNOWLEDGEMENT}

We thank Associate Professor Ruth Vanbaelen, Nihon University, for English language assistance.

\section{REFERENCES}

[1] Kunimatsu, T., Hirai, C. \& Tomii, N., Train rescheduling evaluation from the viewpoint of passengers by microsimulation of train operation and passenger flow. IEEJ Transactions on Industry Applications, 133(7), pp. 756-764, 2013. DOI: 10.1541/ ieejias.133.756. (In Japanese)

[2] Takagi, R., Newly developed simple railway timetable evaluation program Sujic with the new model to deal with re-scheduling. Computers in Railways XIII, (13), pp. 513-520, 2012. DOI: 10.2495/CR120431.

[3] 'Railway diagram recover technology', Ohm-sya, 2010. (In Japanese)

[4] Katori, T., Yamazaki, H. \& Izumi, T., Generating a train rescheduling timetable considering the train routes on tracks and car types. Computers in Railways XIV, (14), pp. 675-685, 2014. DOI: 10.2495/CR140561. 